\title{
Bistatic scattering simulations of circular and linear polarizations over land surface for signals of opportunity reflectometry
}

\author{
Xuerui $\mathrm{Wu}^{1,2}$, Yezhi Song ${ }^{1,2}, \operatorname{Jin}_{\mathrm{Xu}}^{3^{*}} \mathbb{C}$, Zheng Duan ${ }^{4}$ and Shuanggen $\operatorname{Jin}^{1,2}$
}

\begin{abstract}
Signals of Opportunity Reflectometry (SoOp-R) employs the communication system, GNSS (Global Navigation Satellite System) constellation and other potential Signals of Opportunity (SoOp) as the transmitters. In recent years, it has gained increased interests. Several experiments have been carried out, however it is still in the initial development stage. Theoretical predictions of SoOp Reflectometry for land surface parameters detection, such as soil moisture and vegetation biomass, should be carried out simultaneously. Meanwhile, at present less works are paid attention to the polarization study of the polarizations. The first-order radiative transfer equation models are employed here and they are developed according to the wave synthesis technique to get the various polarization combinations. Using the two models as analysis tools, we simulate the bistatic scattering at all potential SoOp Reflectometry bands, i.e., P-, L-, Cand X-band for circular polarizations and linear polarizations. While the original commonly used microwave scattering models are linear polarizations, here we compare the difference. Although the models can simulate bistatic scattering at any incident angles and scattering angles. Four special observation geometry are taken into considerations during the analysis. Using the developed models as tools, the developed models establish the relationship between the land surface parameters (such as soil moisture, soil roughness and vegetation water content, diameters et al.) and bistatic radar cross section. The forward scattering models developed here enables the understanding of the effects of different geophysical parameters and transmitter-receiver observation scenarios on the bisatic scattering at any polarization combinations for any potential SoOP reflectometry bands. Robust retrieval methods for soil moisture and vegetation biomass can benefit from the forward scattering models.
\end{abstract}

Keywords: SoOp Refletometry, GNSS-R, Soil moisture, Vegetation, Polarization, Bistatic scattering

\section{Introduction}

Compared with traditional remote sensing observations by polar-orbiting satellites, GNSS-R technology can provide continuous observation signals in most areas on the earth's surface. It will be a useful supplement to polar-orbiting satellite observations in terms of temporal and spatial resolution and has become the current

\footnotetext{
*Correspondence: jinxu@gdou.edu.cn

${ }^{3}$ Maritime College, Guangdong Ocean University, Zhanjiang 524088,

China

Full list of author information is available at the end of the article
}

international hot research direction (Zavorotny and Gleason 2014; Cardellach and Vey 2016).

The UK-DMC (United Kingdom-Disaster Monitoring Constellation) and TDS-1 (TechDemoSat-1) test satellites were launched for GNSS-R remote sensing research in Year 2004 and 2014, respectively (Gleason and Adjrad 2005; Unwin et al. 2016). In December 2016, CYGNSS (Cyclone Global Navigation Satellite) System) was successfully launched. Its main scientific goal was to measure wind field information on the ocean surface during tropical storms and hurricanes (Ruf et al. 2019; Mayers and Ruf 2019; Crespo et al. 2019). It also provides an important opportunity for land surface parameters and 
cryospheric research (Chew and Small 2018; Morris et al. 2019; Chew et al. 2017). In June 2019, China launched the Bufeng-1 A and B satellites with GNSS-R payload, which is mainly used for sea surface wind field detection (Jing et al. 2019). The GNSS-R space-based payload GNOS II (Global Navigation Satellite System Occultation Sounder II) developed by the National Space Science Center of the Chinese Academy of Sciences is expected to be launched in Year 2022 with the E-star of the FY-3 meteorological satellite (Sun et al. 2017). Although these satellite-borne observation programs are currently mainly used for sea surface parameter monitoring, their development has also opened a door for land surface remote sensing monitoring (Alejandro 2013; Balakhder and Al-Khaldi 2019).

In recent years, SoOp Reflectometry has gained increasing interests (James et al. 2017). This new promising remote sensing technique employs the RF systems (also known as "signals of opportunity" or SoOp) as transmitters. Different from the previous GNSS-R, which mostly use L-band signals of GNSS constellations as transmitted signals, SoOp Reflectomtery employs all potential transmitted signals working at P-band, C-band, $\mathrm{X}$-band and so on. Different frequency bands have different wavelengths, thus results in the various penetration depths. Each frequency bands have their own advantages for geophysical parameters detection. Lower frequency for example P-band, are suitable for root zone soil moisture and forest biomass detection (Yueh et al. 2018). Higher frequency are suitable for snow properties detection (Shah and Yueh 2017). With so many potential free transmitters working at different microwave frequency bands, SoOp Reflectometry provides more and more opportunity for land surface parameters detection. How to dig the wider frequency bands resources and make fully use of them for land surface remote sensing needs researchers more efforts. Scattering mechanisms study is an efficient way for this kind of research and can help demonstrate its feasibility (Kurum et al. 2019; Wu et al. 2014).

As for SoOp Reflectometry, the transmitters and the corresponding receivers form the typical bistatic radar mode. The complexity of the bistatic and changing observation geometry make this technique hard to employ its angle information. Due the much higher scattering amplitudes at specular scattering plane, at present, most researches employ the coherent scattering for soil moisture retrieval using CYGNSS data (Calabia et al. 2020; Kim and Lakshmi 2018; Yan and Huang 2020). As for the ocean surface, the scattering is mostly diffuse scattering (Dong and Jin 2019). However, as for the land surface, some researchers employ the incoherent scattering for soil moisture retrieval (Clarizia et al. 2019; Al-Khaldi and Johnson 2019). With so many signals sources of the transmitters, they provide large amount of observation angles, however, still less work focus on fully dig the angles information for geophysical parameters detection. This limits the development of SoOp Reflectometry. Scattering properties at various observation geometry need though understand. At this stage, bistatic scattering models are needed. Without field measurement data, such as ground-based and airborne-based experiments, theoretical models can provide simulations for various angles combinations, thus illustrating the scattering properties (Wu and Jin 2019, 2020).

To overcome the inosphere effects, most the SoOP transmitters transmit the right hand circular polarization (RHCP) signals. Several experiments have employed linear and circular polarization antennas to collect the reflected signals. In the SMEX02/03 (Soil Moisture Experiments 02/03) airborne GPS-R experiment, the DMR (Delay Map Receiver) with RHCP to collect the direct signals and LHCP (left hand circular polarization) to collect the reflected signals are used (Katzberg et al. 2006). While in the later BAO-Tower experiments, four antennas of vertical polarization, horizontal polarization, RHCP and LHCP are used. Their original hypothesis is to use the polarization ratio to decrease the roughness effects for soil moisture retrieval. However, the results are not as good as they supposed (Zavorotny and Masters 2003). Some researchers employ SIMIGOL (Soil Moisture Interference pattern GNSS Observations at L-band Reflectometer) with vertical polarization antenna and PSMIGOL (the dual-polarization SMIGOL) receivers with horizontal antennas for the geophysical parameters detection (Rodriguez-Alvarez and Camps 2010; AlonsoArroyo and Camps 2014). LEiMON (Land Monitoring with Navigation Signals) experiment employs $\mathrm{RHCH}$ and LHCP for their experiment (Egido and Caparrini 2011). As for the land surface detection, receivers with different polarizations have been carried out, and most the traditional microwave scattering are linear polarizations. However, less work paid attention to the scattering properties of various polarizations.

With the more frequency bands coverage of SoOp Reflectometry, to utilize its angle geometry and polarizations information, we developed microwave scattering models that can accurately understand and describe the microwave scattering mechanism of rough surfaces and vegetation parameters, it is a key mechanism tool for analyzing and interpreting satellite observations, satellite data simulation, satellite data assimilation, development of quantitative inversion algorithms for surface parameters, and new sensor design (Fung 1994).

More details of the model introduction are presented in "Scattering models" section. The corresponding simulations are shown in "Simulations and analysis" 
section. While the conclusion part is given in "Conclusion" section.

\section{Scattering models}

As for our analysis, we employ the first-order radiative transfer equation model to calculate the bare soil and forest scattering properties. In this model, the tall forest canopy is divided into three layers, the crown layer, the trunk layer and the ground layer. As we all know that the commonly used microwave scattering models are either backscattering model for monostatic radar (active microwave scattering model) or the emissivity model for radiometer (passive microwave scattering model). However, we need a bistatic scattering model for GNSS-R and SoOP-R applications, since we know that the transmitters and corresponding receivers of this kind of remote sensing form the typical bistatic radar. In this way, we need modify the commonly used backscattering scattering form of the first order transfer equation model to the bistatic scattering model form. Meanwhile, it should also be noted that to overcome the inospheric effects, the transmitted signals of the GNSS constellation and some of the communication satellite system are circular polarization. However, most of the present microwave scattering models are linear polarizations, we need modify the model and develop ciruclar polarization scattering models. It also be noted that although the payload polarization of the present space-borne CYGNSS and Bufeng- 1 are left hand circular polarizations, their main purpose is oriented for the ocean surface. Nowadays, the space-borne GNSS-R missions for land surface applications are employing the linear polarizations antenna to get the reflected signals. Therefore, our models need to be modified to have the abilities of calculating the circular-linear polarization scattering properties. To get the bistatic scattering model from the traditional backscattering model, we need to convert the BSA (Backward Scatter Alignment) coordinate system to the FSA (Forward Scatter Alignment) coordinate system. "Model description" section shows the model description. The method to transform the models from backscattering form to the bistatic scattering form is presented in "Transform from backscattering form to the bistatic scattering model" section. Polarization coordinate system and the polarization transformation method are presented in. "Polarization coordinate systems" and "Wave synthesis technique" sections. To make the model more clearly, we have given a flowchart in "Model flowchart" section . We have also presented the model validation shown in "Model validation" section.

\section{Model description}

The bistatic Michigan Microwave Canopy Scattering model (short for Bi-MIMICS) is employed for the calculation of the bistatic forest scattering, the first-order fully polarimetric transformation matrix is used (Liang and Pierce 2005). In the Bi-MIMICS model, the canopy are divided into three layers: the crown layer, the trunk layer and the ground layer.

As for the ground part, there are four random rough surface scattering models. The first one is the Kirchhoff model which is under the stationary phase approximation and it is suitable for very rough surface, the second one is the Kirchhoff model under the scalar approximation and it is suited for intermediate scales of roughness, the third one is the small perturbation model and it is suitable for surfaces with short correlation lengths. The above mentioned three models are for discontinuous rough surface. The random rough surface is continuously changing, therefore the forth model of advance equation model is developed and commonly used. For detail complex formula, please see the reference (Ulaby et al. 1988; Chen and $\mathrm{Wu} 2003$ ).

There are eight scattering mechanisms in the model (Liang and Pierce (2005): the direct ground term (DG), the direct crown bistatic scattering term (DC), the crown scattering and ground reflection term $(\mathrm{C}-\mathrm{G})$, the ground reflection and crown scattering term $(\mathrm{G}-\mathrm{C})$, the ground reflection and crown scattering and ground reflection term (G-C-G), the trunk scattering and ground reflection term (T-G) and the ground reflection and trunk scattering term (G-T) and the specular ground term $(\mathrm{S}-\mathrm{G})$. Figure 1 illustrate the above mentioned scattering mechanisms.

\section{Tranform from backscattering form to the bistatic scattering model}

Iterative algorithms are used to solve the radiative transfer equation. Assume that the incident density is $I_{i}\left(\theta_{i}, \varphi_{i}\right)$, the scattered density is $I_{s}\left(\theta_{s}, \varphi_{s}\right)$, the incident zenith angle and azimuth angle are $\theta_{i}, \varphi_{i}$, respectively. While $\theta_{s}, \varphi_{s}$ is the scattered zenith angle and azimuth angle. To simplify the calculation, we assume the incident azimuth angle is $0^{\circ}\left(\varphi_{i}=0^{\circ}\right)$. The observation geometry for the backscattering direction is $\theta_{s}=\theta_{i}, \varphi_{s}=180^{\circ}$, the one for the specular scattering direction is $\theta_{s}=\theta_{i}, \varphi_{s}=0^{\circ}$, while the observation angles for the forward scattering direction is $\theta_{s}=180^{\circ}-\theta_{i}, \varphi_{s}=0^{\circ}$.

After the incident energy is scattered by scatterer, the relationship between the scattering energy intensity and the incident energy intensity can be connected by the transformation matrix $\mathrm{T}$, which is calculated by the extinction matrix and the phase matrix. 


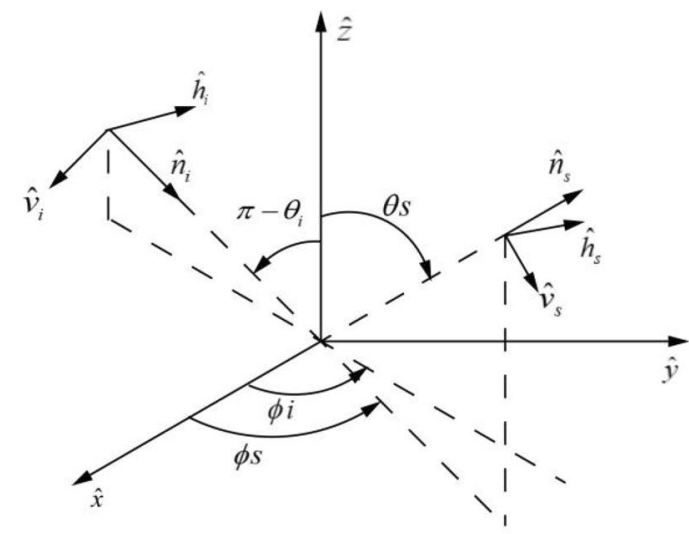

a Geometry for the FSA convention.

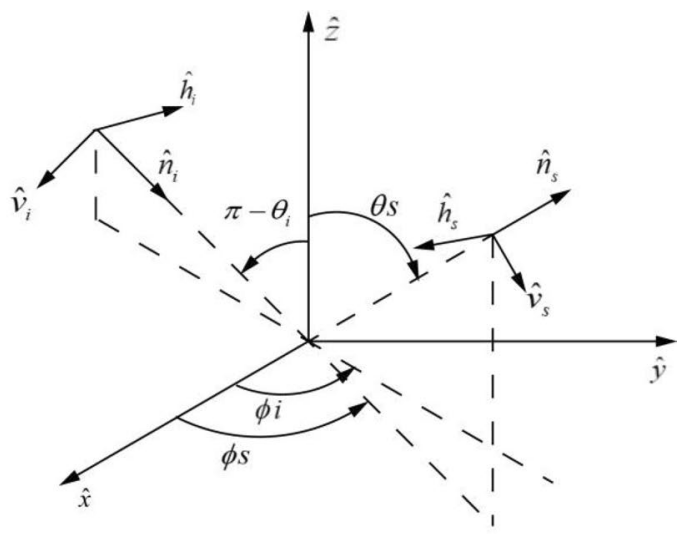

b Geometry for the BSA convention.

Fig. 1 Geometry for the polarization coordinate systems

$$
I_{s}\left(\theta_{s}, \varphi_{s}\right)=T\left(\theta_{s}, \varphi_{s}\right) I_{i}\left(\theta_{i}, \varphi_{i}\right)
$$

The average modified Mueller matrix can be used to calculate the phase matrix and the extinction matrix. For more details and the formulations used to calculate the above mentioned matrices, please see the references (Ulaby et al. 1988; Chen and Wu 2003).

It is noteworthy that the angles should be changed and modified correctly when we transforming the backscattering model to the bistatic scattering model and it is the core contents during the model development. We should modify the original single incident angles to the $\left(\theta_{i}, \varphi_{i}\right)$ to the bistatic form, that is to say, we need to add the scattering angles $\left(\theta_{s}, \varphi_{s}\right)$ in the developed model.

As for the extinction of the crown layer, it include the upward extinction matrix after the scattering by the trunk layer $\left(\theta_{i}=\theta_{s}, \varphi_{i}=\varphi_{s} ; \theta_{s}=\theta_{s}, \varphi_{s}=\varphi_{s}\right)$ and the downward extinction matrix that are not scattered by the trunk layer $\left(\theta_{i}=\pi-\theta_{i}, \varphi_{i}=0 ; \theta_{s}=\pi-\theta_{i}, \varphi_{s}=0\right)$. The extinction matrices in the trunk layer include four kinds: two upwards directions include the one that is scattered by the trunk layer $\theta_{i}=\theta_{s}, \varphi_{i}=\varphi_{s} ; \theta_{s}=\theta_{s}, \varphi_{s}=\varphi_{s}$ and the one does not scatter by the crown layer $\theta_{i}=\theta_{i}, \varphi_{i}=0 ; \theta_{s}=\theta_{i}, \varphi_{s}=0$, and two downwards directions include the one that is scattered by the crown layer $\theta_{i}=\pi-\theta_{s}, \varphi_{i}=\varphi_{s} ; \theta_{s}=\pi-\theta_{s}, \varphi_{s}=\varphi_{s}$ and the other one that does not scattered by the crown layer $\left.\theta_{i}=\pi-\theta_{i}, \varphi_{i}=0 ; \theta_{s}=\pi-\theta_{i}, \varphi_{s}=0\right)$.

As for the calculation of the phase matrix, it includes four kind of observation angles combination, which is shown in the Table 1.
Table 1 Observation angles combinations as for the phase matrix calculation

\begin{tabular}{ll}
\hline The incident direction & Scattering direction \\
\hline$\theta_{i}=\theta_{i}, \varphi_{i}=0$ & $\theta_{S}=\theta_{s}, \varphi_{s}=\varphi_{S}$ \\
$\theta_{i}=\theta_{i}, \varphi_{i}=0$ & $\theta_{S}=\pi-\theta_{s}, \varphi_{S}=\varphi_{s}$ \\
$\theta_{i}=\pi-\theta_{i}, \varphi_{i}=0$ & $\theta_{S}=\theta_{s}, \varphi_{S}=\varphi_{S}$ \\
$\theta_{i}=\pi-\theta_{i}, \varphi_{i}=0$ & $\theta_{S}=\pi-\theta_{s}, \varphi_{S}=\varphi_{S}$ \\
\hline
\end{tabular}

\section{Polarization coordinate systems}

There are commonly two kinds of conventions to define the electromagnetic wave when considering scattering from a scatterer. One is the Forward Scatter Alignment (FSA) convention, the other one is the BackScatter Alignment (BSA) convention. Figure 1 illustrate the geometry for the FSA and BSA conventions. FSA defines the vertical and horizontal unit polarization vectors for the incident and scattered waves and they are equal in the forward scatter direction (Ulaby et al. 1988).

The incident direction and scattering direction are depicted by $\hat{n}_{i}$ and $\hat{n}_{s}$, while $\widehat{v}$ and $\widehat{h}$ are the unit polarization vectors for the vertical and horizontal components, the subscript $i$ and $s$ indicate the incident field and the scattered field. The angles information are expressed by $\left(\theta_{i}, \phi_{i}\right)$ and $\left(\theta_{\mathrm{s}}, \phi_{s}\right)$ for incident and scattered fields.

$$
\begin{aligned}
& \widehat{\mathrm{n}}_{i}=\hat{x} \sin \theta_{i} \cos \phi_{i}+\hat{y} \sin \theta_{i} \sin \phi_{i}+\hat{z} \cos \theta_{i} \\
& \hat{h}_{i}=-\hat{x} \sin \phi_{i}+y \cos \phi_{i}
\end{aligned}
$$




$$
\begin{aligned}
& \widehat{\mathrm{v}}_{i}=\hat{h}_{i} \times \hat{n}_{i}=\hat{x} \cos \theta_{i} \cos \phi_{i}+\hat{y} \cos \theta_{i} \sin \phi_{i}-\hat{z} \sin \theta_{i} \\
& \hat{n}_{\mathrm{s}}=\hat{x} \sin \theta_{s} \cos \phi_{s}+\hat{y} \sin \theta_{s} \sin \phi_{s}+\hat{z} \cos \theta_{s} \\
& \hat{h}_{\mathrm{s}}=-\hat{x} \sin \phi_{s}+\hat{y} \cos \phi_{s} \\
& \widehat{\mathrm{v}}_{s}=\hat{h}_{s} \times \hat{n}_{s}=\hat{x} \cos \theta_{s} \cos \phi_{s}+\hat{y} \cos \theta_{s} \sin \phi_{s}-\hat{z} \sin \theta_{s}
\end{aligned}
$$

As for the BSA convention, it defines the vertical and horizontal unit polarization vectors for the incident and scattered wave and they are equal in the backscatter direction.

$$
\begin{aligned}
& \widehat{\mathrm{n}}_{i}=\hat{x} \sin \theta_{i} \cos \phi_{i}+\hat{y} \sin \theta_{i} \sin \phi_{i}+\hat{z} \cos \theta_{i} \\
& \hat{h}_{i}=-\hat{x} \sin \phi_{i}+y \cos \phi_{i} \\
& \widehat{\mathrm{v}}_{i}=\hat{h}_{i} \times \hat{n}_{i}=\hat{x} \cos \theta_{i} \cos \phi_{i}+\hat{y} \cos \theta_{i} \sin \phi_{i}-\hat{z} \sin \theta_{i} \\
& \hat{n}_{\mathrm{s}}=\hat{x} \sin \theta_{s} \cos \phi_{s}+\hat{y} \sin \theta_{s} \sin \phi_{s}+\hat{z} \cos \theta_{s} \\
& \hat{h}_{\mathrm{s}}=\hat{x} \sin \phi_{s}-\hat{y} \cos \phi_{s} \\
& \widehat{\mathrm{v}}_{s}=-\hat{h}_{s} \times \hat{n}_{s}=\hat{x} \cos \theta_{s} \cos \phi_{s}+\hat{y} \cos \theta_{s} \sin \phi_{s}-\hat{z} \sin \theta_{s}
\end{aligned}
$$

The only difference between the BSA and FSA convention is that $\hat{h}_{\mathrm{s}}$ is replaced with $-\hat{h}_{\mathrm{s}}$. The BSA convention has been the standard used in the area of radar polarimetry. To get the various polarization combination using the wave synthesis technique, we do the polarization coordinate systems conversion accordingly.

\section{Wave synthesis technique}

To get the circular polarization properties, here we employ the wave synthesis technique to make the scattering models can calculate various polarization combination (Ulaby and Elachi 1990).

$$
\sigma_{r_{t}}\left(\psi_{r}, \chi_{r}, \psi_{t}, \chi_{t}\right)=4 \pi \tilde{Y}_{m}^{r} M_{m} Y_{m}^{t}
$$

$M_{m}$ is the modified Mueller matrix, $Y_{m}$ is the modified stokes vectors, where the upper subscript $r$ and $t$ indicate the polarization of transmitted and received signals. These depend on the variables of orientation $\psi$ and ellipticity $\chi$, respectively. The polarization of an electromagnetic wave may be described in terms of the ellipticity diagram shown in Fig. 2. It is sufficient to use the angles of $\psi$ and $\chi$ to specify the polarization completely.

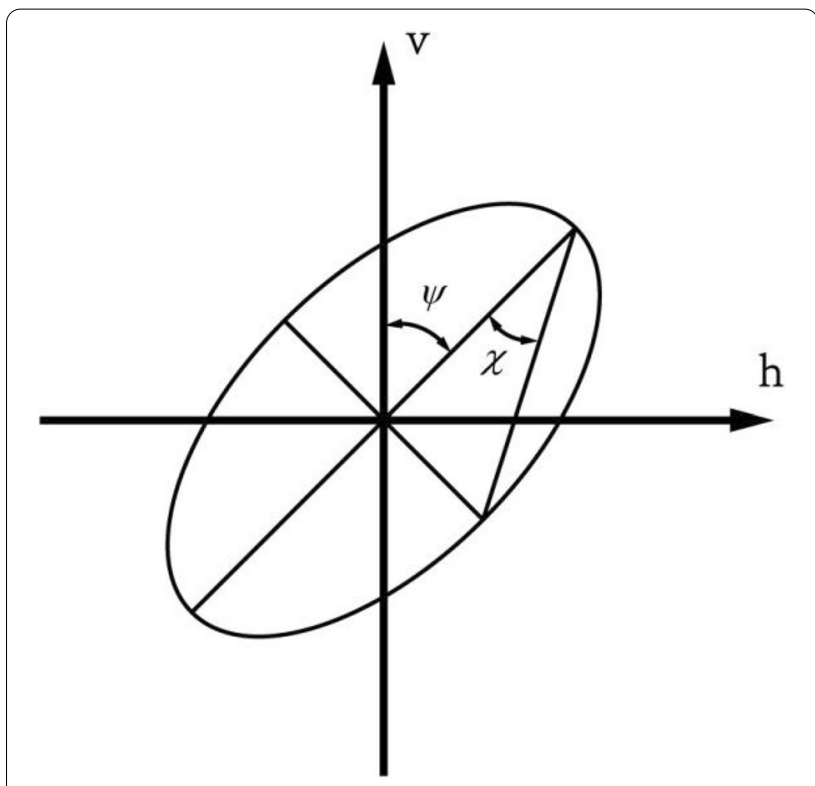

Fig. 2 Polarization of an electromagnetic wave

It should be noted that the modified Stokes vector is an alternative description of wave polarization. While the modified Stokes vectors can be defined like the following and it is a function of the angles $\psi$ and $\chi$. For different polarization, the settings of the modified Stokes are like Table 2.

$$
Y_{m}=\left[\begin{array}{c}
\frac{1}{2}(1+\cos 2 \psi \cos 2 \chi) \\
\frac{1}{2}(1-\cos 2 \psi \cos 2 \chi) \\
\sin 2 \psi \cos 2 \chi \\
\sin 2 \chi
\end{array}\right]
$$

\section{Model flowchart}

Figure 3 shows the model flowchart. As for the random surface scattering model, Soil temperature, soil moisture and texture and surface roughness are the soil physical input parameters. They are combined with the satellite geometry for model inputs. Using the wave synthesis technique, the model can get various polarization combinations for different kind of soil. As for the vegetation part, the vegetation physical parameters are the important part of the model inputs, such as vegetation water content, density and diameters, these information are also combined with the satellite geometry for the inputs of the forest scattering model. By employing the wave synthesis technique, we can get bistatic scattering of bare soil and vegetation with various polarization combinations. 
Table 2 Modified Stokes vectors for various polarizations

\begin{tabular}{|c|c|c|c|c|c|c|}
\hline & V pol & $\mathrm{H}$ pol & LHCP pol & RHCP pol & $+45^{\circ} \mathrm{pol}$ & $-45^{\circ} \mathrm{pol}$ \\
\hline Modified Stokes vector & {$\left[\begin{array}{l}1 \\
0 \\
0 \\
0\end{array}\right]$} & {$\left[\begin{array}{l}0 \\
1 \\
0 \\
0\end{array}\right]$} & {$\left[\begin{array}{c}0.5 \\
0.5 \\
0 \\
1\end{array}\right]$} & {$\left[\begin{array}{c}0.5 \\
0.5 \\
0 \\
-1\end{array}\right]$} & {$\left[\begin{array}{c}0.5 \\
0.5 \\
-1 \\
0\end{array}\right]$} & {$\left[\begin{array}{c}0.5 \\
0.5 \\
1 \\
0\end{array}\right]$} \\
\hline
\end{tabular}

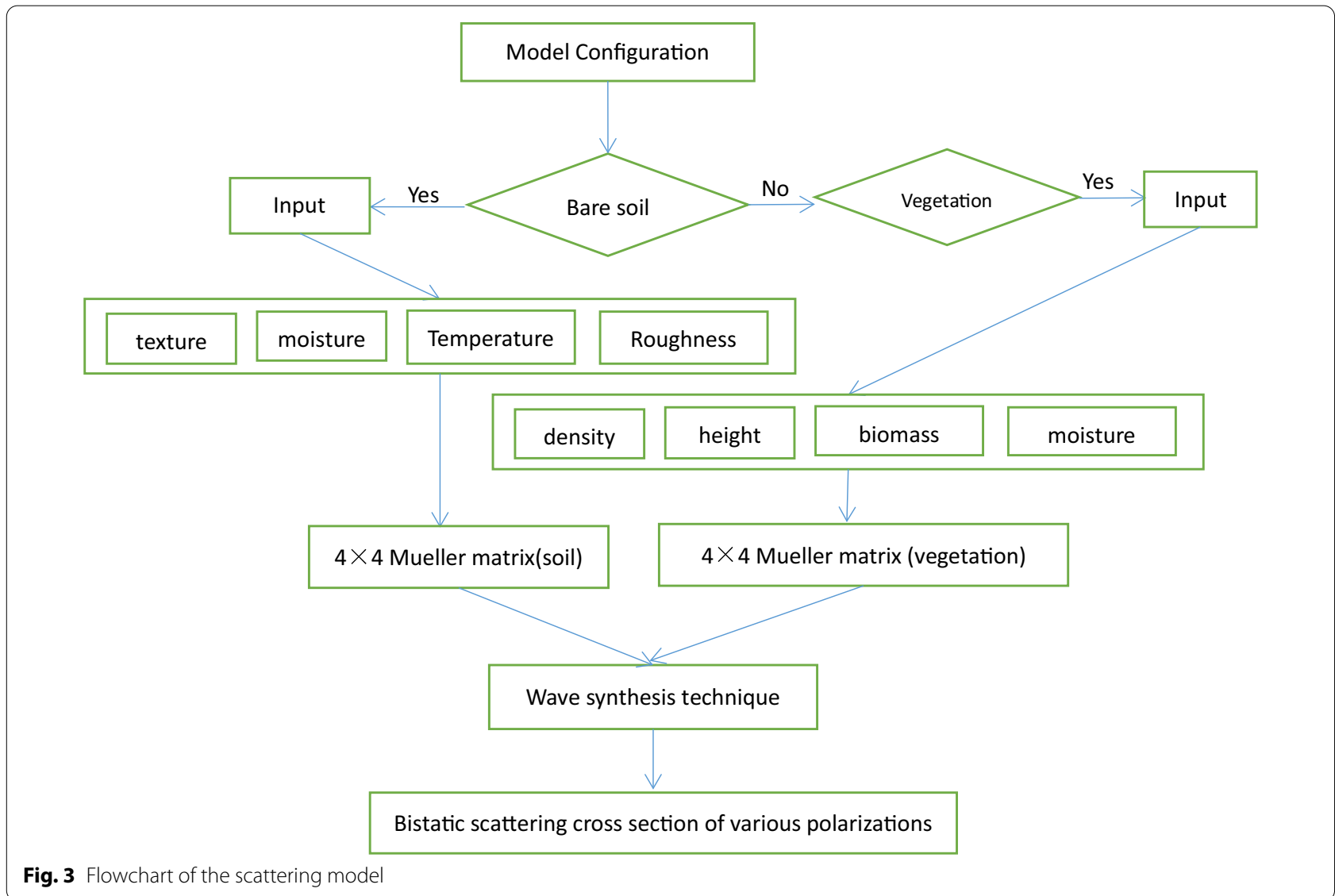

\section{Model validation}

Due to the lack of the bistatic radar measurement data, the above mentioned models cannot be validated with measurement data. However, we know the models of backscattering form have been validated and commonly used (Liang and Pierce 2005). Therefore, we configure our models to the back scattering form by setting the elliptical and ellipticity angle. And not surprisingly, they produce the same results as the original backscattering model. This indicates our model is an efficient model for SoOp Reflectometry study, which is a typical bistatic radar form.

As for the polarization validation, we have employed the wave synthesis technique to get the various polarization combinations, as we know the original model is the linear form, we have set the modified Stokes vectors to the linear form and compare the results with the original model, and not surprisingly, they produce the same results. In this way, we validate the polarization ability of the model and we assume that the models calculations for various polarization combination is correct. Of course, we await for the measurement validation in the future.

\section{Simulations and analysis}

Bare soil simulations

As for the random rough surface simulations, here we simulate the different scattering properties at all potential SoOp Reflectometry frequency bands: P-band(0.48Ghz), L-band (1.48Ghz), C-band (4.75Ghz) 
and X-band (10Ghz). We also simulate the scattering properties at various polarization, such as RR, LR, VR, $\mathrm{HR},+45^{\circ} \mathrm{R}$ and $-45^{\circ} \mathrm{R}$. For the polarization notations, the first letter is the received polarization, the second letter is the transmit polarization. To do comparison, we also simulate the linear polarization properties. Here four kinds of observation geometry is carried out. The incident angle and the scattering angle are presented in Fig. 4, under its definition, the observation geometry of $\left\{\theta_{s}=\theta_{i}, \varphi_{s}=180^{\circ}\right\}$ indicates backscattering; $\left\{\theta_{s}=\theta_{i}, \varphi_{s}=0^{\circ}\right\}$ stands for specular scattering; $\left\{\theta_{s}=\theta_{i}, \varphi_{s}=90^{\circ}\right\}$ denotes the perpendicular scattering. While $\left\{\theta_{i}=30^{\circ}, \theta_{s}=\left(10^{\circ}-85^{\circ}\right), \varphi_{s}=120^{\circ}\right\}$ is one illustration of bistatic scattering. These four special

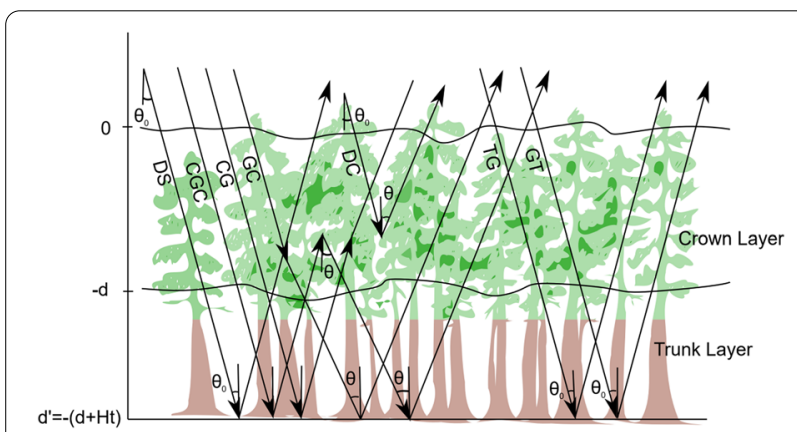

Fig. 4 Scattering mechanisms in the first-order Bi-mimics model, including the GCG, CG, DC, GC, GT, DG, and TG terms. The SG term is not shown. The crown layer depth is $Z 1=d$ and the trunk layer depth $\mathrm{Z} 2=\mathrm{Ht}$ observation geometries are employed and analyzed as the following.

Figure 5 presents the linear and circular polarization random rough surface scattering cross section versus scattering angle for $\mathrm{P}-, \mathrm{L}-, \mathrm{C}$ - and X-band at backscattering plane. From the point of scattering amplitude, as for linear polarization, the cross polarization, i.e., $\mathrm{VH}$ polarization (Fig. 5c), is the smallest one. As for circular polarization, RR polarization (Fig. $5 \mathrm{~d}$ ) the smallest one among the circular polarizations as shown in Fig. $5 \mathrm{e}-\mathrm{i}$. It should be noted that there are scattering notches as for VV polarization (Fig. 5b) and VR polarization due to the Brewster angle (Fig. 5f), which is very useful for the vegetation correction during soil moisture retrieval. It is worth mentioning that the VV polarization means the transmitted polarization and the received polarizations are all vertical polarization, while VR means the transmitted signals are vertical polarization and the received polarization is right hand circular polarization. In Fig. 5, different frequency bands are simulated, as for the same scattering observation geometry and polarization, $\mathrm{X}$-band is the smallest one, $\mathrm{C}$-band is larger than X-band. While P-band and L-band is the largest one and their scattering properties are very near the same, which is due to the close frequency bands. When the backscattering angle is at lower and moderate range, the differences for these four frequency bands are more obvious, except for P-band and L-band, the scattering properties are almost the same for higher backscattering angle.

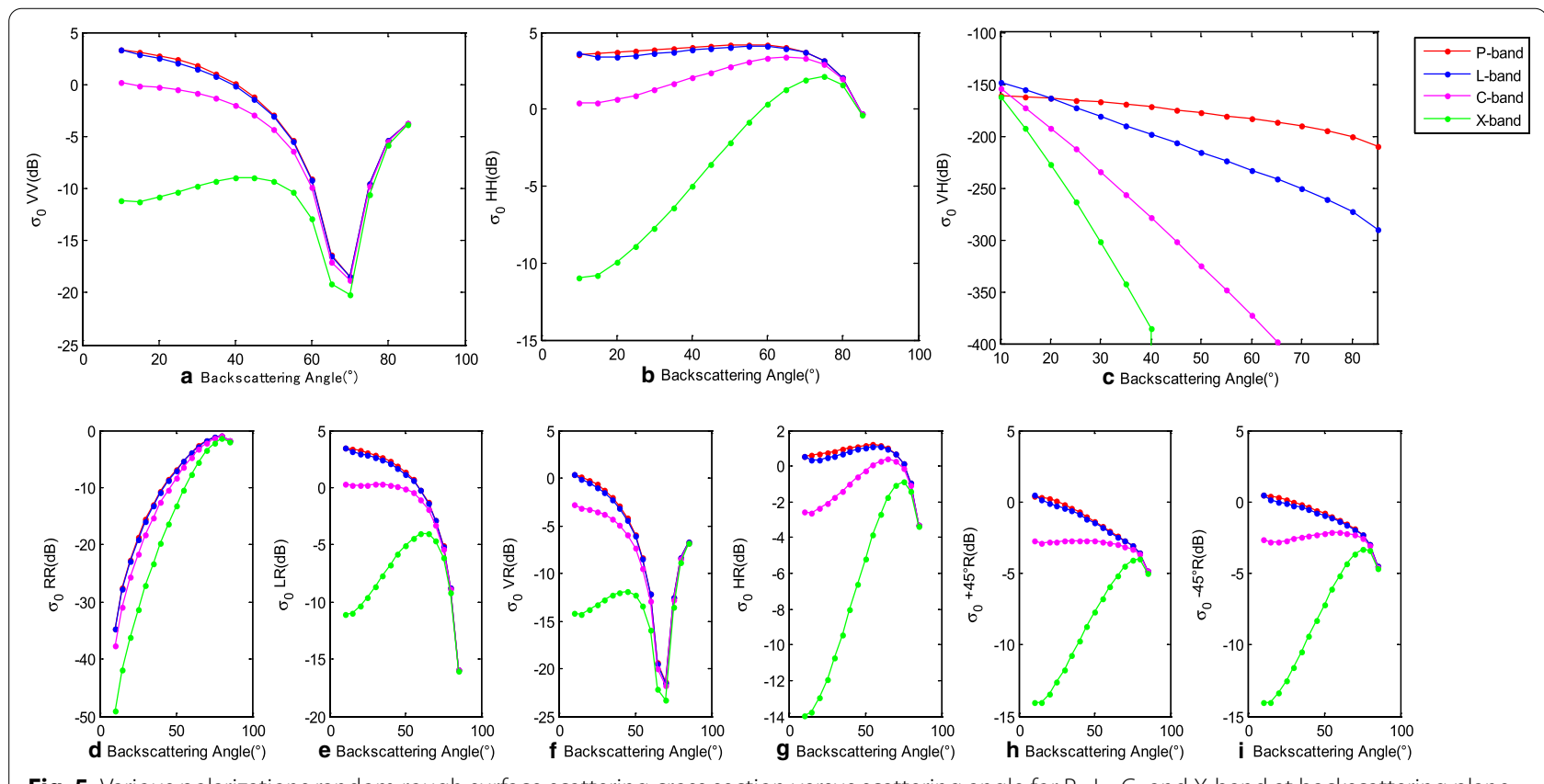

Fig. 5 Various polarizations random rough surface scattering cross section versus scattering angle for $P$-, L-, C- and X-band at backscattering plane 
Figure 6 presents various polarizations random rough surface scattering cross section versus scattering angle for P-, L-, C- and X-band at $\theta_{\mathrm{i}}=30^{\circ}, \varphi_{s}=120^{\circ}$. From the simulations, we can see that the bistatic scattering at this observation geometry is very small for all polarizations. This means the diffuse scattering is very small. We can also see that the scattering notches that appear in the VV polarization and VR polarization as shown in Fig. 3 do not exist. The scattering trends for all polarizations are almost the same. For the same observation geometry, the magnitude of the scattering amplitude for different frequency bands is $\mathrm{P}-, \mathrm{L}-, \mathrm{C}$ - and X-band in turn. For smaller observation scattering angle, the differences between the four frequencies are smaller. As the observation scattering angle increase, the difference between these four frequency bands are becoming more and more obvious.

Figure 7 presents various polarizations random rough surface scattering cross section versus scattering angle for P-, L-, C- and X-band at perpendicular plane. From the point of scattering amplitude, as for linear polarizations, cross polarization, i.e., VH polarization (Fig. 6c) is the much smaller than the co-polarizations at VV and $\mathrm{HH}$ polarization. As for circular polarization, RR pol (the transmitted and received polarizations are all right hand circular polarizations) is smaller than the scattering at LR, VR, HR, $+45^{\circ} \mathrm{R}$ and $-45^{\circ} \mathrm{R}$, whose scattering amplitude is the same as VV and $\mathrm{HH}$ polarization. As for VV and VR polarization, there are scattering notches due to the Brewster angle. As for X-band scattering at LR, VR,
$\mathrm{HR},+45^{\circ} \mathrm{R}$ and $-45^{\circ} \mathrm{R}$, there are scattering notches at smaller incident angle. For all the polarizations, scattering at X-band is the smallest, while scattering at P-band and L-band is almost the same and their scattering values are larger than $\mathrm{C}$-band and $\mathrm{X}$-band. The differences of scattering values at various polarizations (except $\mathrm{VH}$ polarization) at smaller and moderate incidence angles are more obvious, while the scattering differences for different frequency bands at larger incident angles are almost the same. As for $\mathrm{VH}$ polarization, scattering values at smaller incidence angles are almost the same for different frequency bands, but the differences become more obvious at larger incidence angles.

Figure 8 presents various polarizations random rough surface scattering cross section versus scattering angle for $\mathrm{P}-, \mathrm{L}-, \mathrm{C}$ - and $\mathrm{X}$-band at specular plane. There is no specular scattering values at $\mathrm{VH}$ polarization. From the simulations as shown in Fig. 8, we can see that the specular scattering have the largest scattering values among the different observation geometry (From Figs. 5, 6, 7). Due to the Brewster angle, there are scattering notches at VV and VR polarizations as expected. It should be noted that the scattering amplitude for different frequency bands are opposite compared to the simulations as shown in Figs. 5, 6 , scattering at X-band is larger than the other frequency bands, scattering at P-band and L-band is smaller than that at X-band and C-band.

From Figs. 5, 6, 7, 8, we simulate the random surface scattering at four different observation geometry. Specular scattering has the largest scattering values. Due to the

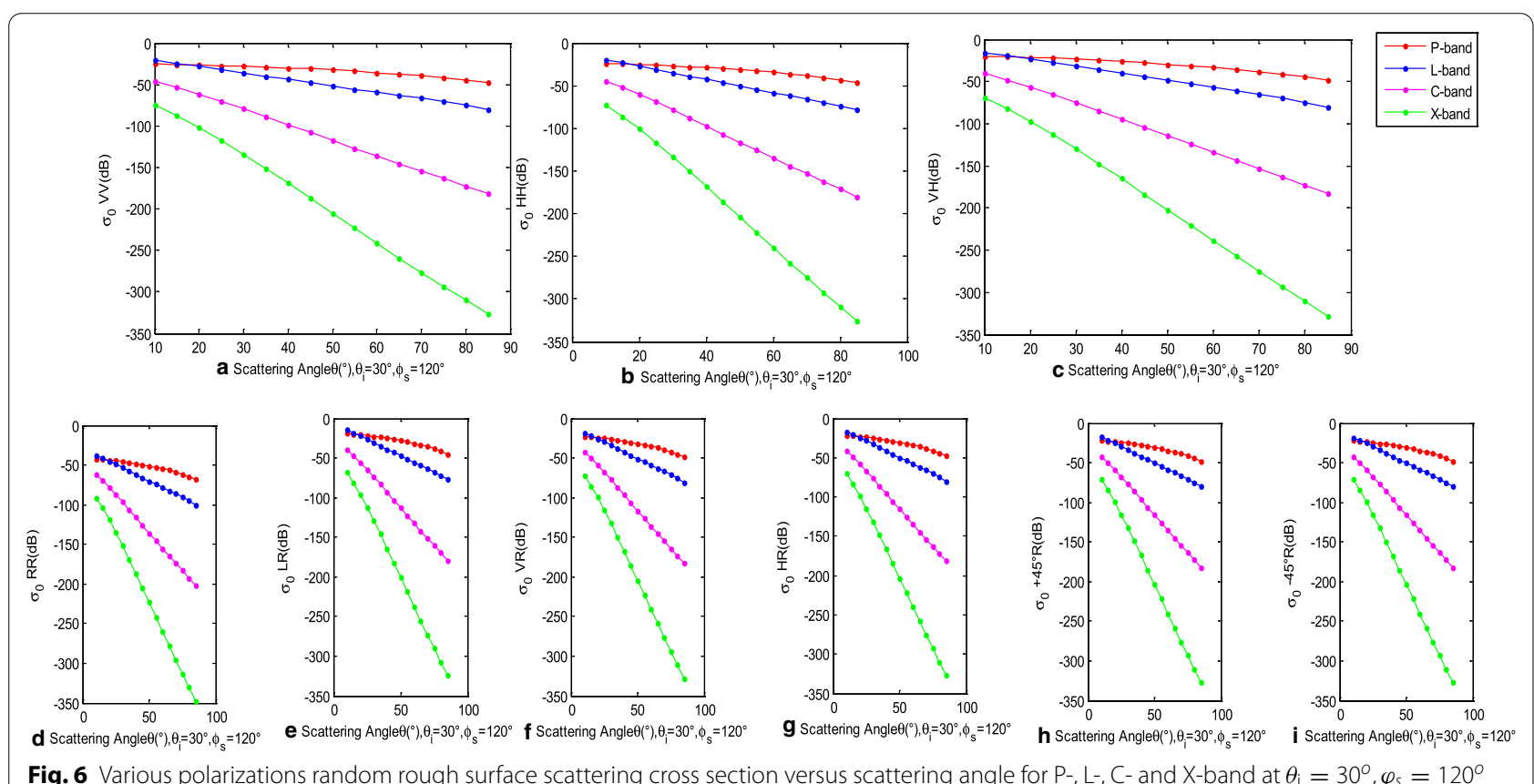



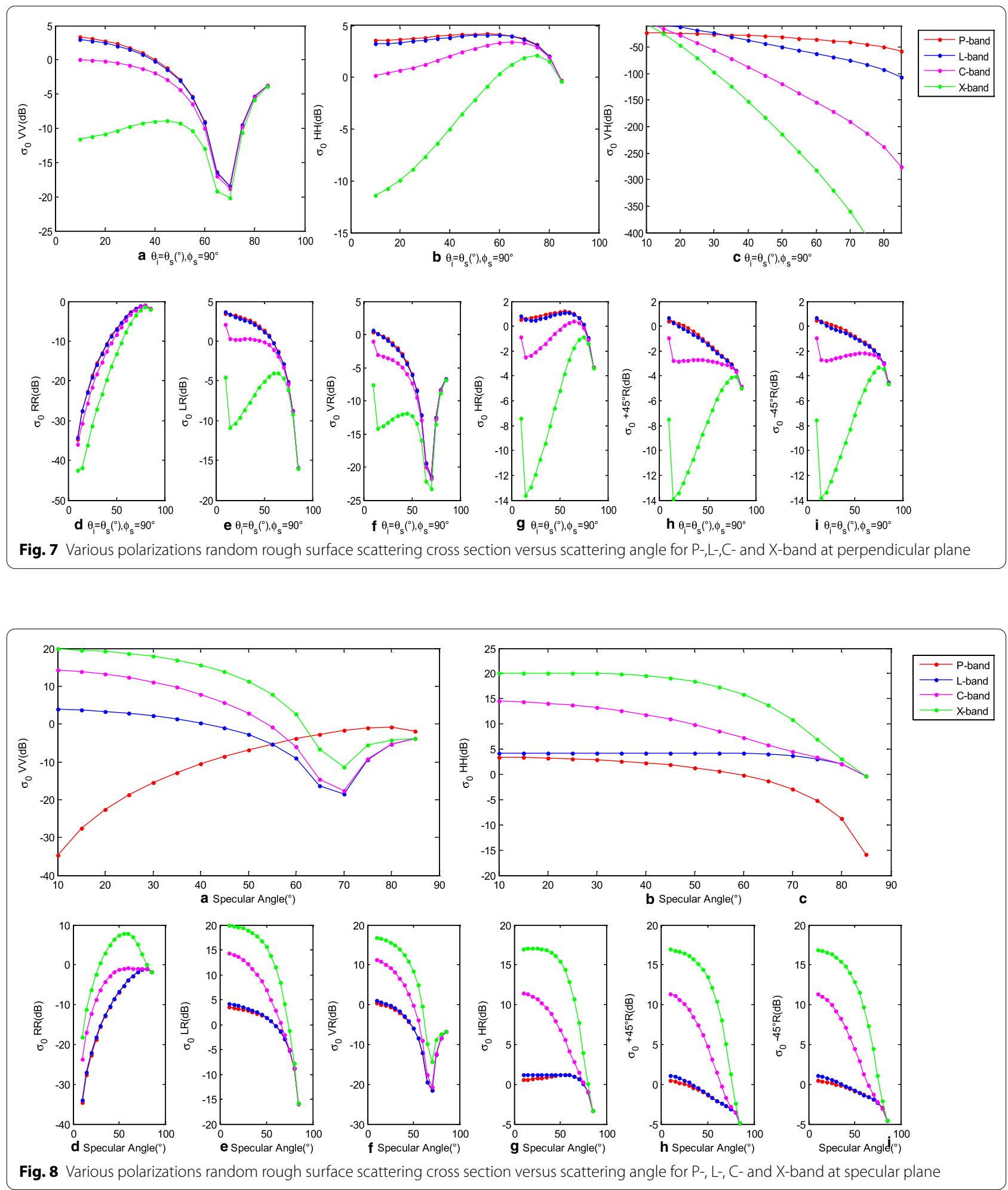

Brewster angle, there are scattering notches at specular, perpendicular and backscattering plane. As for linear polarizations, scattering values at cross-polarization
(VH polarization) has the smallest scattering values. As for circular polarization, scattering values at $R R$ polarization is the smallest one. Except for specular 
scattering, scattering values decrease as the frequency bands increase for the same the observation geometry. However, the trends change opposite as for the specular scattering.

\section{Vegetation scattering}

As for the vegetation (aspen as presented in reference is an illustration (Ulaby et al. 1988) scattering, we also simulate the different scattering properties for four different observation geometry. From the above simulations as shown in Figs. 5, 6, 7, 8, scattering properties at $\pm 45^{\circ} \mathrm{R}$ is almost the same. Therefore, in this part, we ignore the simulations at these two polarizations and focus on the scattering properties at linear polarization and RR, LR, VR and HR polarization.

Figure 9 presents the canopy scattering cross section versus scattering angle from aspen for $\mathrm{L}-, \mathrm{C}$ - and $\mathrm{X}$-bands at backscattering plane. As for linear polarization, cross polarization, i.e., $\mathrm{VH}$ polarization, has lower scattering values than the co-polarization. As for circular polarization, scattering values at $R R$ polarization has lower scattering values than that at the LR, VR and HR polarization. For different polarizations, the relationship for P-, L-, C- and X-bands are complex, which is due to the different volume scattering in the canopy layer. For $\mathrm{VH}$ polarization and $\mathrm{RR}$ polarization, the scattering properties at different frequency bands are more obvious than the other polarizations.
Figure 10 presents the canopy scattering cross section versus scattering angle from aspen for $\mathrm{L}-, \mathrm{C}-$-, and $\mathrm{X}$-bands at $\theta_{\mathrm{i}}=30^{\circ}, \varphi_{s}=120^{\circ}$. For this observation geometry, it happens diffuse scattering. We can see that the most obvious appearance is that there is a scattering peak at $\theta_{\mathrm{i}}=\theta_{\mathrm{s}}=30^{\circ}$, which is due to the strong scattering caused by the trunk. The scattering amplitude for various polarizations are almost the same.

Figure 11 presents the canopy scattering cross section versus scattering angle from aspen for $\mathrm{L}_{-}, \mathrm{C}-$, and $\mathrm{X}$-bands at perpendicular plane. The scattering amplitude for different polarizations are almost the same. The scattering trends for X-band are more different than the other frequency bands especially at lower incident angles. For the other frequency bands, the scattering properties are very close. But as the scattering angle varies, the scattering trends for $\mathrm{P}-, \mathrm{L}-, \mathrm{C}$ - and $\mathrm{X}$-band are very different. Therefore, observation geometry is a very important effect that affect the scattering values.

Figure 12 shows the canopy scattering cross section versus scattering angle from aspen for L-, C-, and $\mathrm{X}$-bands at specular plane. Compared with Figs. 9, 10, 11, the specular scattering has larger scattering values than the other observation geometry. From the simulations, we can see that scattering values at $\mathrm{VH}$ polarization has the smallest values among all the various polarizations. For the same observation geometry and polarizations, we can see that the scattering values increase as the frequency bands increase. In general, the scattering values

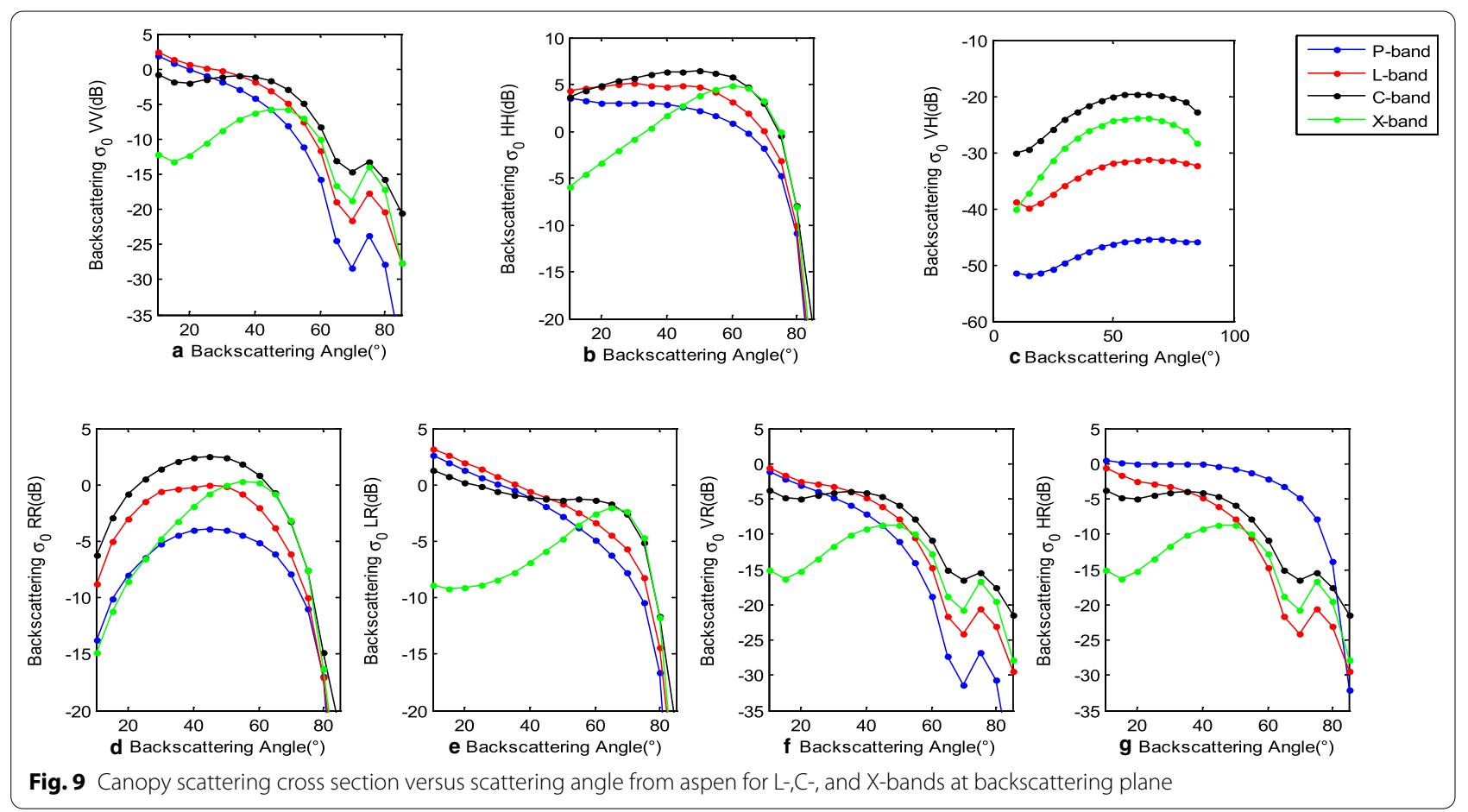



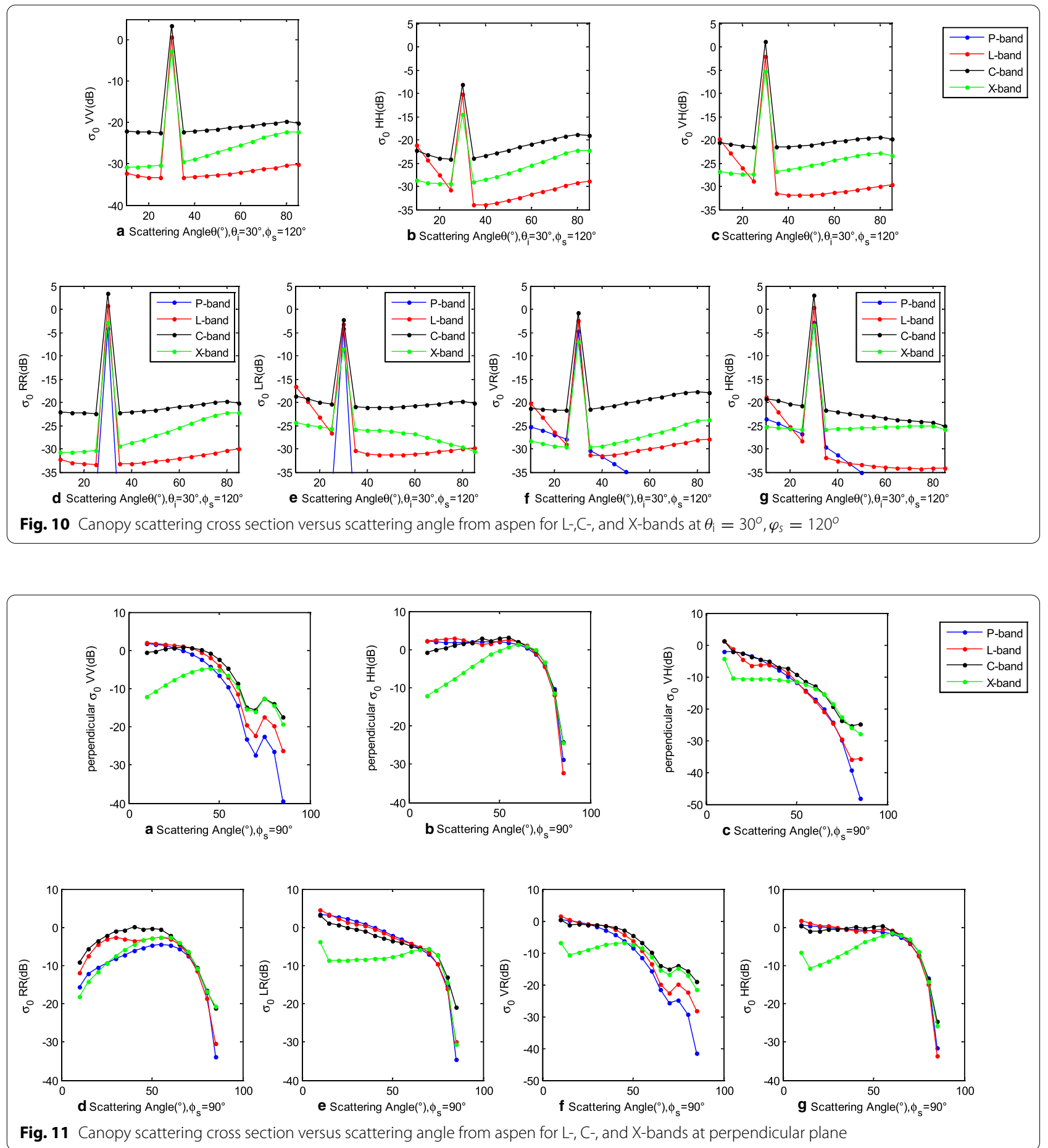

decrease sharply for larger specular angles except for RR polarization. The specular scattering firstly increase and then decrease for RR polarization.

From the simulations as presented from Figs. 9, 10, 11 , we illustrate the bistatic scattering for four different observation geometry. We can see that scattering values at the specular direction has larger values than the other observation geometry. Different from the random rough surface scattering properties, the scattering trends and relationship for vegetation canopy is more complex. This is due the different scattering mechanisms that dominate the total scattering. 


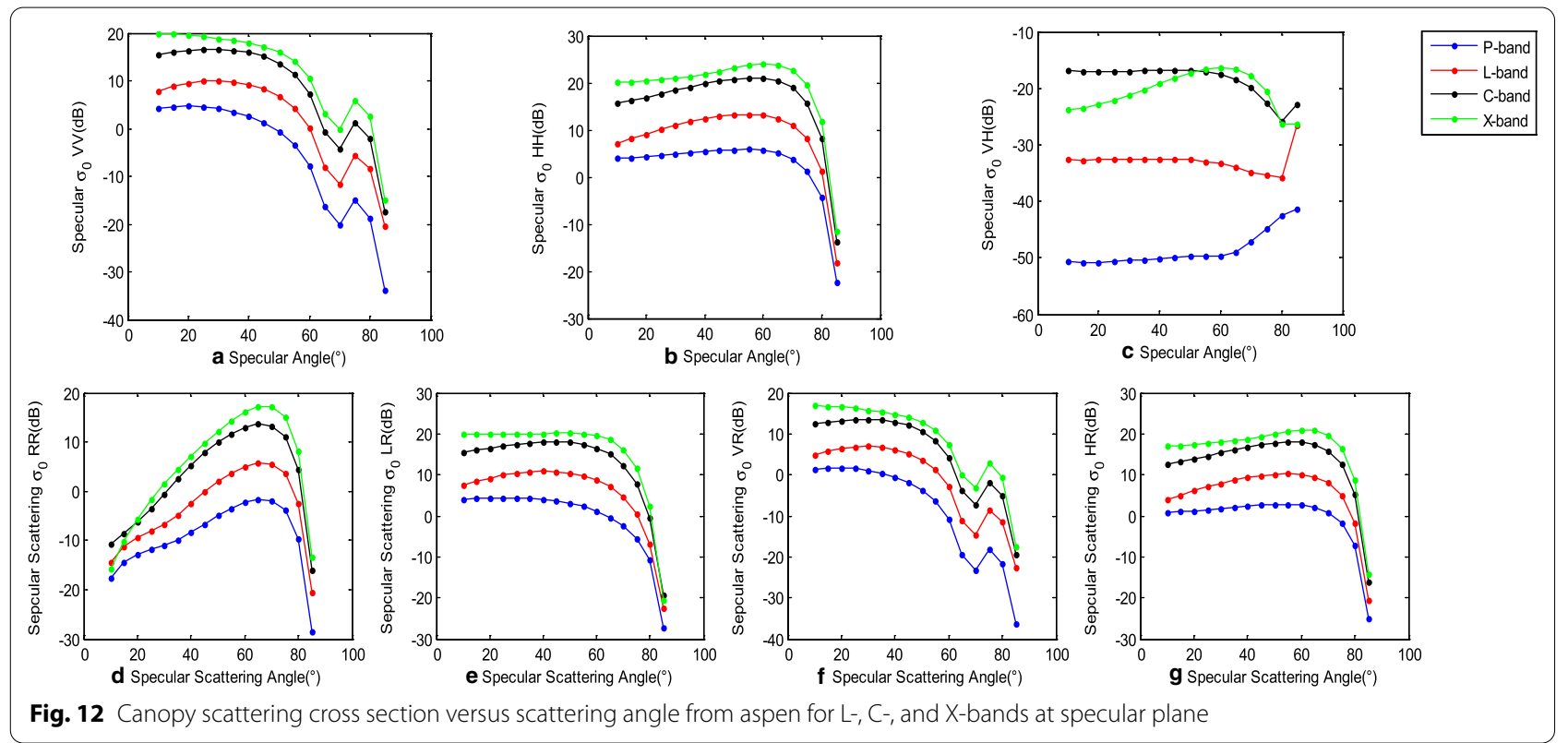

In this part, we can see that our developed models have the ability to simulate the bistatic scattering at various polarizations and different frequency bands. This will be an efficient microwave tool for SoOp Refelctometry study.

\section{Conclusion}

There is an increased interest in using all potential reflected Signals of Opportunity for geophysical parameters, such as soil moisture and vegetation biomass study in recent years. Although several experimental and theoretical studies have demonstrated the potential of such applications, there are still many unknowns that need be explored. Here we employed the random rough surface scattering models and the vegetation scattering models to relate the bare soil, vegetation parameters and system observation geometry with the bistatic scattering cross sections. The scattering models are thus a critical tool for data explanation, future mission and experimental campaigns design. By employing the wave synthesis technique, the models can calculate the bistatic scattering at any polarization combination for all potential SoOP Reflectometry frequency bands. Bistatic scattering at linear polarization and circular polarization scattering properties are simulated and compared for analysis in this paper. Although the results are not validated by experimental data, the model simulations predict the bistatic scattering properties suitable for SoOP Reflectometry applications in terms of all polarizations and angular combinations. As for random rough surface scattering, there are notches at VV and VR polarizations, which is caused by the
Brewster angle, it should be noted that these notches only exist at backscattering, specular and perpendicular plane. The notches angles in these polarizations can be a good efficient information for bare soil retrieval. As considering for the vegetation canopy scattering, the notches angles disappear, which is due to the complex volume scattering and reflection in the different canopy layers. How to use the notches information to improve the soil moisture retrieval accuracy and get rid of vegetation effects are a promising direction in the future SoOp Reflectometry development. Al tough scattering at RR polarization is lower than the other circular polarization, it can provide the polarization ratio to improve the geophysical parameters retrieval. This advancement of theoretical simulations for bare soil and vegetation can predict the bistatic scattering properties at any polarizations for all potential SoOp Reflectometry bands, thus benefiting the future land surface SoOp Reflectometry development.

\section{Acknowledgements}

This research was funded by the National Natural Science Foundation of China (No. 42061057 \&NO.41501384\&42074042). The authors would be very grateful for the suggestions given by Dr. Andres Calabia in School of Remote Sensing and Geomatics Engineering, Nanjing University of Information Science and Technology.

\section{Authors' contributions}

XRW: idea proposal, conceptualization, and original draft; YGS: idea proposal and suggestions; JX: figure plot; ZD: suggestions; SGJ: funding. All authors read and approved the final manuscript.

\section{Availability of data and materials}

This paper is about a theoretical model. The inputs for the models are from the previous published papers which are shown in the corresponding references part. 


\section{Declarations}

\section{Competing interests}

The authors declare no conflict of interest.

\section{Author details}

1 Shanghai Astronomical Observatory, Chinese Academy of Sciences, Shanghai 200030, China. ${ }^{2}$ University of Chinese Academy of Sciences, Beijing, China.

${ }^{3}$ Maritime College, Guangdong Ocean University, Zhanjiang 524088, China.

${ }^{4}$ Department of Physical Geography and Ecosystem Science, Lund University, Lund, Sweden.

Received: 7 December 2020 Accepted: 20 March 2021

Published online: 01 April 2021

References

Alejandro E (2013) GNSS reflectometry for land remote sensing applications.

Al-Khaldi MM, Johnson JT (2019) Time-Series Retrieval of Soil Moisture Using CYGNSS. IEEE Transact Geosci Remote Sens. 57:1-10

Alonso-Arroyo A, Camps A (2014) Improving the accuracy of soil moisture retrievals using the phase difference of the dual-polarization GNSS-R interference patterns. IEEE Geosci Remote Sens Lett. 11:2090-2094

Balakhder AM, Al-Khaldi MM (2019) On the coherency of ocean and land surface specular scattering for GNSS-R and signals of opportunity systems. IEEE Transact Geoence Remote Sens. 99:1-11

Calabia A, Molina I, Jin S (2020) Soil moisture content from GNSS Reflectometry using dielectric permittivity from fresnel reflection coefficients. Remote Sens 12:122

Cardellach E, Vey S. Foreword to the Special Issue on GNSS Reflectometry. IEEE J Select Topics Appl Earth Observat Remote Sens. 2016: 9(10); 4519-4524.

Chen K, Wu T (2003) Emission of rough surfaces calculated by the integral equation method with comparison to three-dimensional moment method simulations. Geosci Remote Sens IEEE Transact. 41:90-101

Chew CC, Small EE (2018) Soil moisture sensing using spaceborne GNSS reflections: Comparison of CYGNSS reflectivity to SMAP soil moisture. Geophys Res Lett. 45:4049-4057

Chew C, Reader JT, Small E (2018) CYGNSS data map flood inundation during the 2017 Atlantic hurricane season. Sci Rep. 8(1):1-8

Clarizia MP, Pierdicca N, Costantini F, Floury N (2019) Analysis of CYGNSS data for soil moisture retrieval. IEEE J Selected Topics Appl Earth Observat Remote Sens 12(7):2227-2235

Crespo JA, Posselt DJ, Asharaf S (2019) CYGNSS surface heat flux product development. Remote Sens. 11(19):2294

Dong ZN, Jin SG (2019) Evaluation of spaceborne GNSS-R retrieved ocean surface wind speed with multiple datasets. Remote Sens. 11(23):2747

Egido A, Caparrini M (2011) LEiMON Land Monitoring With Navigation Signals. ESA/ESTEC, Noordwijk, Netherlands, Final Rep. ESA Contract22117/08/ NL/AF.

Fung AK (1994) Microwave scattering and emission model and their applications.

Gleason S, Adjrad M (2005) Sensing ocean, ice and land reflected signals from space: results from the UK-DMC GPS reflectometry experiment.

James G, Lin YC, Benjamin N (2017) Recent results on soil moisture remote sensing recent results on soil moisture remote sensing using P-band signals of opportunity.

Jing C, Niu X, Duan C, Lu F, Yang X (2019) Sea surface wind speed retrieval from the first chinese gnss-r mission: Technique and preliminary results. Remote Sens 11:3013

Katzberg SJ, Torres O, Grant MS, Masters D (2006) Utilizing calibrated GPS reflected signals to estimate soil reflectivity and dielectric constant: results from SMEX02. Remote Sens Environ 100:17-28
Kim H, Lakshmi V (2018) Use of Cyclone Global Navigation Satellite System (CyGNSS) observations for estimation of soil moisture. Geophys Res Lett. 45(16):8272-8282

Kurum M, Deshpande M, Joseph AT, O'Neill PE, Lang RH, Eroglu O (2019) SCoBi-Veg: a generalized bistatic scattering model of reflectometry from vegetation for signals of opportunity applications. IEEE Transact Geosci Remote Sens. 57(2):1049-1068

Liang P, Pierce LE (2005) Radiative transfer model for microwave bistatic scattering from forest canopies. Geosci Remote Sens IEEE Transact. 43(11):2470-2483

Mayers D, Ruf CS (2019) Tropical Cyclone Center Fix using CYGNSS Winds. J Appl Meteor Climatol. 58:1993-2003

Morris M, Chew C, Reager JT, Shaw R, Zuffada C (2019) A novel approach to monitoring wetland dynamics using CYGNSS: everglades case study. Remote Sens Environ. 233:111417

Rodriguez-Alvarez N, Camps A (2010) Land geophysical parameters retrieval using the interference pattern GNSS-R technique. Geosci Remote Sens 49(1):71-84

Ruf C, Asharaf S, Balasubramaniam R, Gleason S, Lang T, McKague D, Twigg D, Waliser D (2019) In-orbit performance of the constellation of CYGNSS hurricane satellites. Bull Amer Meteor Soc. 100:2009-2023

Shah R, Yueh S (2017) Remote sensing of terrestrial snow using signals of opportunity applications at VHF/UHF bands. Geosci Remote Sens Sympos. 14:309-313

Sun Y, Liu C, Du Q, Wang X, Liu C. Global Navigation Satellite System Occultation Sounder II (GNOS II). In Proceedings of the IGARSS IEEE International Geoscience \& Remote Sensing Symposium, Fort Worth, TX, USA. 2017.

Ulaby FT, Elachi C (1990) Radar polarimetry for geoscience applications. Artech House Inc, Norwood, p 376

Ulaby FT, Sarabandi K, Mcdonald KY, Whitt M, Dobson MC (1988) Michigan Microwave Canopy Scattering model (MIMICS). Univ Michigan Ann Arbor Tech Rep. 11:1223-1253

Unwin M, Jales P, Tye J, Gommenginger C, Foti G, Rosello J (2016) Spaceborne GNSS-reflectometry on TechDemoSat-1: early mission operations and exploitation. IEEE J Sel TOp Appl Earth Obs Remote Sens. 9(10):4525-4539

Wu XR, Jin SG (2014) GNSS-Reflectometry: Forest canopies polarization scattering properties and modeling. Adv Space Res. 54(5):863-870

Wu XR, Jin SG (2019) A simulation study of GNSS-R polarimetric scattering from the bare soil surface based on the AIEM model. Adv Meteorol. https://doi.org/10.1155/2019/3647473

Wu XR, Jin SG (2020) Models and theoretical analysis of SoOP circular polarization bistatic scattering for random rough surfaces. Remote Sens. 12(9):1506

Yan Q, Huang W (2020) Pan-tropical soil moisture mapping based on a threelayer model from CYGNSS GNSS-R data. Remote Sens Environ 247:111944

Yueh SH, Xu X,Shah R, Margulis S, Elder K. P-Band Signals of Opportunity for Remote Sensing of Root Zone Soil Moisture. IGARSS 2018-2018 IEEE International Geoscience and Remote Sensing Symposium, Valencia, 2018, 1403-1406.

Zavorotny VU, Gleason S (2014) Tutorial on remote sensing using GNSS bistatic radar of opportunity. Geosci Remote Sens Magazine 2(4):8-45

Zavorotny V, Masters D (2003) Seasonal polarimetric measurements of soil moisture using tower-based gps bistatic radar. Geoscience and Remote Sensing Symposium. IGARSS '03. Proceedings. IEEE International.

\section{Publisher's Note}

Springer Nature remains neutral with regard to jurisdictional claims in published maps and institutional affiliations. 